\title{
MISQ Archivist
}

\section{A Configural Approach to Coordinating Expertise in Software Development Teams}

\author{
Srinivas Kudaravalli, Samer Faraj, and Steven L. Johnson
}

\begin{abstract}
Despite the recognition of how important expertise coordination is to the performance of software development teams, understanding of how expertise is coordinated in practice is limited. We adopt a configural approach to develop a theoretical model of expertise coordination that differentiates between design collaboration and technical collaboration. We propose that neither a strictly centralized, top-down model nor a largely decentralized approach is superior. Our model is tested in a field study of 71 software development teams. We conclude that because design work addresses illstructured problems with diverse potential solutions, decentralization of design collaboration can lead to greater coordination success and reduced team conflict. Conversely, technical work benefits from centralized collaboration. We find that task knowledge tacitness strengthens these relationships between collaboration configuration and coordination outcomes and that team conflict mediates the relationships. Our findings underline the need to differentiate between technical and design collaboration and point to the importance of certain configurations in reducing team conflict and increasing coordination success in software development teams. This paper opens up new research avenues to explore the collaborative mechanisms underlying knowledge team performance.
\end{abstract}

Keywords: Software development, software teams, expertise coordination, configuration, centralization, knowledge tacitness, team conflict, coordination success 